\title{
Report of an international postsymposium to the 23rd EASD Annual Meeting on Molecular Genetics of Diabetes Mellitus
}

\author{
H.Zühlke and R. Walther \\ Institute of Biochemistry, University of Greifswald, GDR
}

A postsymposium of the 23rd Annual Meeting of the European Association for the Study of Diabetes (EASD) was held in RostockWarnemünde, GDR, September 19th-21st, 1987. The meeting was organised by the Institute of Biochemistry of the Ernst-MoritzArndt-University Greifswald, GDR, and supported by the Ministry of Health of the GDR.

At the meeting 64 scientists from 15 countries took part. Experimental results and future prospects of the following topics were discussed:

1. The insulin gene, its structure and control of expression

2. Polymorphisms related to diabetes mellitus

3. Regulation of expression of transferred genes in mammalian cells

Concerning the first topic new information was presented regarding the insulin gene itself and various regulatory events at different levels of expression. Dr. Ohlsson (Umea, Sweden) reported on DNA elements and transacting factors involved in the regulation of the transcription of the rat insulin gene I. A report by Dr. Welsh and coworkers (Uppsala, Sweden) dealt with the influence of dexamethason, calcium ions and phorbol ester on the regulation of insulin gene transcription. An interesting paper was given by Dr. Hoirris-Nielsen (Gentofte, Denmark) who demonstrated that the cytokine interleukin-1 affects (pro)insulin biosynthesis at the pre- and post-translational level in a dose-dependent manner.

Introducing the second topic Dr. Mayr (Vienna, Austria) gave an overview about polymorphisms and diseases, in particular diabetes mellitus. There are a number of possibilities for a connection between a genetic marker and disease. These include a direct involvement of the marker in the disease, linkage of a marker gene with disease genes, or association between markers and diseases. Several examples were critically discussed regarding their medical importance.

Dr. Neumer et al. (Greifswald, GDR) gave a report about a restriction fragment length polymorphism (RFLP) close to the human insulin gene on chromosome 11 and its possible relation to diabetes mellitus in a GDR population. In a report given by Dr. Kanazawa et al. (Tokyo, Japan) the ethnic differences in the incidence of the diabetes types as well as consequences for the evaluation of insulin gene RFLP's were presented comparing Japanese and Caucasians. Similar conclusions were drawn by Dr. Hitman (London, UK) presenting data on RFLP's of the HLA D-region from British Caucasoids and South Indian Type 1 (insulin-dependent) diabetic subjects. It also became evident that HLA-related polymorphisms are of particular interest in studying genetic susceptibility in Type 1 diabetes.

This was strengthened by Dr. Michelsen (Gentofte, Denmark) who presented results of a HLA D-related RFLP in a Danish and Swedish population. Another interesting polymorphism concerning Type 2 (non-insulin-dependent) diabetes mellitus was introduced by Dr. Galton (London, UK) who has studied genetic variants of the insulin receptor. Summarising this topic, it became clear that the investigation of RFLPS may become, at some future time, clinically relevant to a better characterisation of diabetic patients.

Within the third topic new trends in studying the expression of transferred wildtype and mutant insulin genes, as well as first tendencies with regard to gene therapy were presented. Using several plasmids containing preproinsulin genes with varying lengths of the connecting peptide coding sequence Dr. Docherty (Birmingham, UK) demonstrated the connection between the primary structure of the insulin gene and the post-translational processing mechanism of proinsulin to insulin. Within the plasmid used by Dr. Walther and co-workers (Greifswald, GDR) the insulin gene is under control of the mouse metallothionein-I-promotor. Following the transfer of the plasmid into nonpancreatic mammalian cells the formation of immunoreactive insulin in the presence and absence of heavy metal ions was reported on.

In the next paper Dr. Selden (Boston, Mass, USA) presented a model for a somatic cell gene therapy system termed "transkaryotic implantation". Fibroblast cells of a clonal line stable expressing insulin were implanted at various locations into mice and the secreted insulin was monitored in the serum. Dr. Jami (Paris, France) reported on the expression of the human insulin gene on transgenic mice. This approach allows examination of the expression of the introduced gene through all stages of development and also location of the integration site of the introduced gene within the genom. Dr. Höppner (Hamburg, FRG) presented some information about the molecular mechanisms by which glucose and related carbohydrates exert their regulatory role on important cellular proteins.

\section{Dr. H.Zühlke}

Institute of Biochemistry

University of Greifswald

Rubenowstraße 3

DDR-2200 Greifswald

GDR 